\title{
From the Cochrane Library: Emollients and Moisturizers for Eczema
}

\author{
Jarett Anderson $^{\mathrm{a}}$ Jalal Maghfour ${ }^{\mathrm{b}}$ Austin Hamp ${ }^{\mathrm{a}}$ Austin Christensen ${ }^{\mathrm{a}}$ \\ Robert P. Dellavalle ${ }^{c}$ \\ ${ }^{a}$ Arizona College of Osteopathic Medicine, Midwestern University, Glendale, AZ, USA; ${ }^{b}$ Department of Dermatology, \\ Henry Ford Hospital, Detroit, MI, USA; 'Department of Dermatology, University of Colorado Anschutz Medical \\ Campus, Aurora, CO, USA
}

\section{Keywords}

Atopic dermatitis · Eczema · Moisturizers · Emollient ·

Topical corticosteroids $\cdot$ Cochrane review

\section{Dear Editor,}

Although moisturizers are widely used for the management of atopic dermatitis $(\mathrm{AD})$, evidence regarding their efficacy and safety remains largely unexplored. For this reason, a systematic Cochrane review, "Emollients and Moisturisers for Eczema" [1], was performed. The use of moisturizers in skin care remains an important topic, particularly as prolonged mask-wearing during the COVID-19 pandemic has shown to increase the incidence of skin barrier damage amongst healthcare workers and the general population [2].

In this review, 77 randomized controlled trials (RCTs) were analyzed and included 6,603 participants with mildto-moderate eczema. The majority of RCTs $(n=29)$ evaluated moisturizers' efficacy relative to another moisturizer, with the remaining RCTs comparing moisturizers against the following: (1) placebo or vehicle $(n=15),(2)$ no treatment $(n=7)$, or (3) active treatment, such as corticosteroids or immunomodulators $(n=15)$. Fifteen RCTs also compared a combination of a moisturizer with active treatment versus moisturizers only. The primary outcomes measured in this review included (1) partici- pants' self-assessment of disease severity from baseline and (2) participants' satisfaction and adverse events. Clinically significant results with moderate high-quality evidence are summarized in Table 1.

Overall, moisturizers achieved a significant reduction in investigator-assessed disease severity scores $(n=12$ RCTs, 1,281 participants, standardized mean difference $-1.04,95 \%$ CI -1.57 to -0.51 ; high-quality evidence) and fewer flares ( $n=6$ RCTs; RR 0.33, 95\% CI 0.17-0.62; moderate-quality evidence), without demonstrating a difference in adverse events ( $n=10$ RCT; RR $1.03,95 \%$ CI $0.82-$ 1.30; moderate-quality evidence). The systematic review highlights the beneficial effects of moisturizers in the management of $\mathrm{AD}$, particularly when combined with topical corticosteroids. However, current evidence supporting the utility of moisturizers for eczema remains low to moderate $[1,3]$.

Importantly, Van Zuuren et al. [1] were unable to conclude which moisturizers were most appropriate for specific areas of the body, duration of disease, and were largely unable to specify which moisturizers were superior to one another. Further research evaluating the comparative efficacy of moisturizers is of clinical importance as it has the potential to greatly benefit patients, particularly as new information is discovered. Skin barrier damage, which is affected by the use of face masks that are often karger@karger.com

(c) 2021 S. Karger AG, Basel

www.karger.com/drm
Correspondence to:

Robert P. Dellavalle, robert.dellavalle@ucdenver.edu 
Table 1. Moisturizer comparison with respective outcome, comparisons, risk ratio, confidence interval, number of studies, and quality of evidence

\begin{tabular}{|c|c|c|c|c|}
\hline Outcomes & Comparison & $\begin{array}{l}\text { Relative risk and confidence } \\
\text { interval }\end{array}$ & $\begin{array}{l}\text { Number of studies and } \\
\text { participants }\end{array}$ & $\begin{array}{l}\text { Quality of } \\
\text { evidence }\end{array}$ \\
\hline $\begin{array}{l}\text { Change from baseline in disease severity } \\
\text { according to investigators }{ }^{\mathrm{a}}\end{array}$ & Atopiclair vs. vehicle & RR $4.51,95 \%$ CI $2.19-9.29$ & 390 participants, 3 RCTs & Moderate \\
\hline Number of participants who experienced flares & Atopiclair vs. vehicle & RR $0.18,95 \%$ CI $0.11-0.31$ & 397 participants, 3 RCTs & Moderate \\
\hline $\begin{array}{l}\text { Change from baseline in disease severity } \\
\text { according to participants }{ }^{\mathrm{b}}\end{array}$ & $\begin{array}{l}\text { Vehicle treatment + daily moisturizer compared to fluticasone } \\
\text { propionate twice weekly + daily moisturizer }\end{array}$ & RR $0.45,95 \%$ CI $0.34-0.59$ & 348 participants, 1 RCT & High \\
\hline Number of participants who experienced flares & $\begin{array}{l}\text { Vehicle treatment }+ \text { daily moisturizer compared to fluticasone } \\
\text { propionate twice weekly + daily moisturizer }\end{array}$ & RR $2.17,95 \%$ CI $1.51-3.11$ & 718 participants, 4 RCTs & Moderate \\
\hline $\begin{array}{l}\text { Change from baseline in disease severity } \\
\text { according to investigators }^{c}\end{array}$ & Urea-containing moisturizer vs. vehicle, placebo, or nothing & RR $1.40,95 \%$ CI $1.14-1.71$ & 129 participants, 1 RCT & Moderate \\
\hline $\begin{array}{l}\text { Number of participants reporting an adverse } \\
\text { event }\end{array}$ & Urea-containing moisturizer vs. vehicle, placebo, or nothing & RR $1.65,95 \%$ CI $1.16-2.34$ & 129 participants, 1 RCT & Moderate \\
\hline $\begin{array}{l}\text { Change from baseline in disease severity } \\
\text { according to participants }{ }^{\mathrm{d}}\end{array}$ & $\begin{array}{l}\text { Glycerin/glycerol-containing moisturizer vs. vehicle, placebo, } \\
\text { or nothing }\end{array}$ & RR $1.22,95 \%$ CI $1.01-1.48$ & 134 participants, 1 RCT & Moderate \\
\hline Number of participants who experienced flares & All moisturizers vs. placebo, vehicle, or nothing & RR $0.33,95 \%$ CI $0.17-0.62$ & 607 participants, 6 RCTs & Moderate \\
\hline
\end{tabular}

required during the COVID-19 pandemic, has been shown to increase the risk for eczema. Moisturizers have the ability to blunt the effects of the skin barrier damage caused by these masks [2].

Of note, regular skin care is just one of the many aspects central to the maintenance and management of AD. In a recently published study [4], 6 "obligatory" guideline-oriented preventive measures were identified and measured in the management of eczema. While over $90 \%$ of participants reported to be using some form of skin care, only $13.9 \%$ of patients adhered to all 6 measures; patients' level of education was found to be highly predictive of their adherence to these measures [5], highlighting the importance for further implementation and education in managing the disease. The RCTs analyzed by this Cochrane review had variable methodology and often did not report adherence to or implementation of these other guideline-oriented measures, such as refraining from smoking in the living space, relaxation techniques, and avoidance of skin irritants. Thus, it is unclear whether variable adherence to these other guideline-oriented measures contributed to the results provided by these RCTs.

Establishing recommendation guidelines for the use of emollients will likely stem from clinical comparative efficacy. Although a recently published report has demonstrated the feasibility of recruiting patients with eczema in a primary care setting, randomly assigning them to a moisturizer regimen and maintaining observers blinded to allocation for a period of 52 weeks [6], only one RCT is currently assessing the comparative long-term efficacy and safety of various emollients [4], highlighting the need for additional research.

\section{Key Message}

Emollients appear effective in alleviating symptoms of atopic dermatitis particularly when combined with corticosteroids. There is insufficient evidence to support the use of one emollient over another.

\section{Conflict of Interest Statement}

Dr. Dellavalle is a Joint Coordinating Editor for Cochrane Skin, a Dermatology Section Editor for UpToDate, a Social Media Editor for the Journal of the American Academy of Dermatology (JAAD), and a Podcast Editor for the Journal of Investigative Dermatology (JID). He is a Coordinating Editor representative on Cochrane Council.

\section{Funding Sources}

There were no funding sources.

\section{Author Contributions}

J.A. and J.M. significantly contributed to manuscript writing. A.H. created a summary table, while A.C. aided in manuscript revisions. R.P.D. guided us throughout manuscript writing and revisions. 


\section{References}

1 Van Zuuren EJ, Fedorowicz Z, Christensen R, Lavrijsen A, Arents BWM. Emollients and moisturisers for eczema. Cochrane Database Syst Rev. 2017;2(2):CD012119.

2 Wan X, Lu Q, Sun D, Wu H, Jiang G. Skin barrier damage due to prolonged mask use among healthcare workers and the general population during the COVID-19 pandemic: a prospective cross-sectional survey in China. Dermatology. 2021:1-8. Published online July 8.
3 Van Zuuren EJ, Fedorowicz Z, Arents BWM. Emollients and moisturizers for eczema: abridged Cochrane systematic review including GRADE assessments. Br J Dermatol. 2017;177(5):1256-71.

4 Bhanot A, Huntley A, Ridd MJ. Adverse events from emollient use in eczema: a restricted review of published data. Dermatol Ther (Heidelb). 2019;9(2):193-208.
5 Zyriax B-C, Augustin M, Abeck F, Mohr N, Kirsten N, Langenbruch A. Adherence to guideline-oriented preventive measures in patients with atopic dermatitis in Germany. Dermatology. 2021:1-6. Published online June 25.

6 Ridd MJ, Wells S, Edwards L, Santer M, MacNeill S, Sanderson E, et al. Best emollients for eczema (BEE) - comparing four types of emollients in children with eczema: protocol for randomised trial and nested qualitative study. BMJ Open. 2019;9(11):e033387. 